\title{
Nonlinear Soliton Propagation in a Few Mode Optical Fibre
}

\author{
Naoise Mac Suibhne $^{1, \mathrm{a}}$, Regan Watts ${ }^{2}$, Stylianos Sygletos ${ }^{1, \mathrm{~b}}$, Fatima C Garcia Gunning ${ }^{1, \mathrm{~b}}$, Lars \\ Grüner-Nielsen ${ }^{3}$, Andrew D. Ellis ${ }^{1, b}$ \\ ${ }^{1}$ Photonic Systems Group, Tyndall National Institute, Lee Maltings, Cork, Ireland \\ also with ${ }^{a}$ Dept. Electronic Engineering and ${ }^{b}$ Dept Physics, University College Cork, Ireland \\ ${ }^{2}$ School of Electronic Engineering, Dublin City University, Ireland \\ ${ }^{3}$ OFSDenmark, Priorparken 680, 2605 Brøndby, Denmark
}

\begin{abstract}
We experimentally demonstrate adiabatic soliton propagation in the fundamental mode of a few mode optical fibre and more complex behaviour in a higher order mode, indicating that the impact of nonlinearities differs for each mode.
\end{abstract}

\section{INTRODUCTION}

The continued demand on communication systems has historically led to technologies that allow greater capacity in optical networks. More recently the research community has predicted that the capacity limit for single mode optical fibre (SMF) is within sight [1]. Advances in coherent detection, digital signal processing (DSP) and advanced modulation formats are aiming to avoid the capacity crunch in the short to medium term. The impending capacity limit has also served as a catalyst to look beyond SMF with renewed interest on the topic of multimode optical fibres (MMF). In particular few-mode fibres (FMF), which can support higher optical power than SMF due to their larger effective area whilst being less sensitive to typical MMF effects, such as modal crosstalk. This interest has lead to promising experimental demonstrations of spatial mode multiplexing and demultiplexing [2]; theoretical work has also been undertaken on the feasibility and constraints of such systems $[3,4]$.

Numerical models have been proposed for nonlinear transmission in FMF, where only intra-modal nonlinearities and linear mode coupling are considered [3]. Consistent with this approach it has been indicated that solitons may exist in individual modes [5,6]. However, Marcuse [7] has studied similar systems, indicating that nonlinear mode coupling may also occur, and experimental MMF systems demonstrate inter-mode nonlinearities such as self focusing and the abrupt onset of nonlinearity, inconsistent with the simpler models [8]. To the best of our knowledge, the impact of nonlinearity on propagation (particularly in the case of FMF) has yet to be observed experimentally.

In this paper we experimentally investigate the feasibility of FMF transmission systems by studying, for the first time to our knowledge, nonlinear propagation in two modes. We observed that the fundamental mode

The authors would like to acknowledge Science Foundation Ireland under grant number 06/IN/I969 and the EU $7^{\text {th }}$ Framework Programme under grant agreement 228033 (MODE-GAP). exhibits soliton-like behavior, agreeing well with numerical modeling based on the conventional assumptions, but with the higher order mode the behaviour is more complex.

\section{EXPERIMENTS}

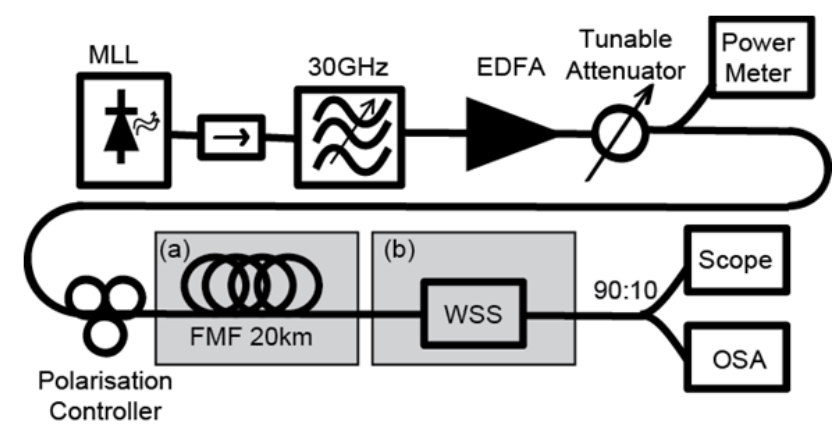

Fig. 1. Experimental setup.

The step index FMF used was $20 \mathrm{~km}$ long and supported four LP modes at 1551nm. The input and output of the fibre were spliced to SMF pigtails using a conventional fusion splicer, which aligned the claddings before splicing. This way, the slight core mismatch enabled the $\mathrm{LP}_{01}$ and $\mathrm{LP}_{02}$ modes to be excited simultaneously with chromatic dispersions of $21.1 \mathrm{ps} / \mathrm{nm} / \mathrm{km}$ and $17.5 \mathrm{ps} / \mathrm{nm} / \mathrm{km}$ and a differential group delay of $2.95 \mathrm{~ns} / \mathrm{km}$. The coupling and splice losses were estimated to be about 6 to $10 \mathrm{~dB}$. Therefore, assuming these values, if a single short pulse is launched to the input fibre, the output should contain two pulses (one for each mode) 58.6ns apart. In order to analyse these two modes, as shown in Fig. 1, a filtered actively modelocked laser (MLL) with a repetition rate of $10 \mathrm{MHz}$ was used as a source of low chirp to generate 23ps pulses allowing the nonlinear impulse response of the modes to be captured.

In order to study nonlinear propagation, an EDFA was added to generate high peak powers which also added dispersion to the transmitter, and therefore careful tuning of the filter was required.

The temporal intensity and phase of the FMF input and output pulses were measured using a photonic frequency discriminator technique employing a programmable wavelength selective switch (WSS) to generate a linear ramp filter to differentiate the temporal field, and the resulting temporal components were resolved using an Agilent sampling scope. More details on the technique 
can be found in [9]. Selected measurements were calibrated using a FROG technique [10], which measured an input pulse width of 23.8ps against 23.1ps from the frequency discriminator. This $\sim 23.4 \mathrm{ps}$ pulse width produces a time-bandwidth product of $\sim 0.66$, confirming that residual dispersion in the amplified pulse source remains. The output of the fibre was monitored with both an optical spectrum analyzer (OSA) and a high-speed optical oscilloscope $(50 \mathrm{GHz})$. The MLL and the scope were both triggered by the same external clock to ensure synchronisation.

\section{RESULTS AND DiscuSSION}
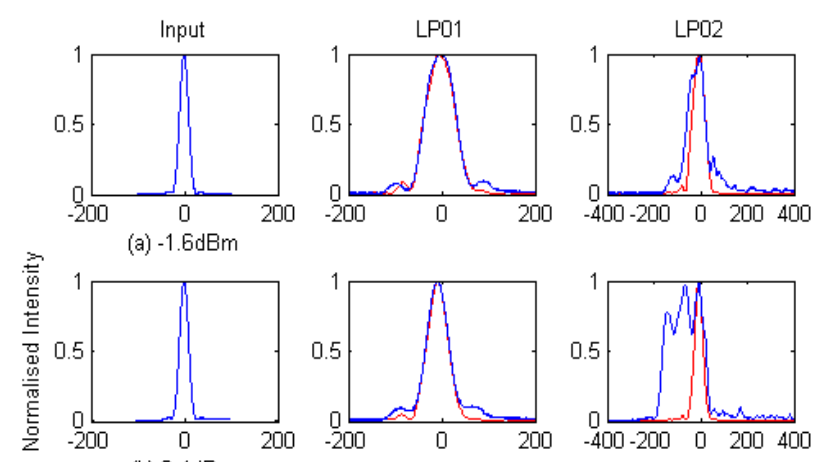

(b) $3.4 \mathrm{dBm}$
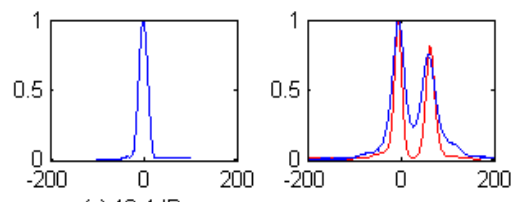

(c) $10.4 \mathrm{dBm}$
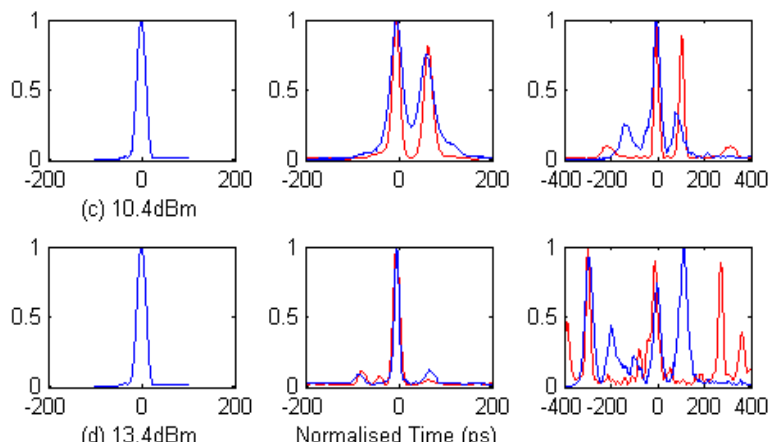

Fig. 2. Scope pulses for three input average power levels of about (a) $-1.6 \mathrm{dBm}$, (b) $3.4 \mathrm{dBm}$, (c) $10.4 \mathrm{dBm}$ and (d) $13.4 \mathrm{dBm}$. Blue are experimental results while red are from the numerical model.

Fig. 2 shows a selection of results for input pulses (left) with different input power levels (a to d), and the output pulses for $\mathrm{LP}_{01}$ and $\mathrm{LP}_{02}$ modes respectively all using the frequency discriminator technique. The two modes were observed to be 58ns apart (not shown) and this is consistent with the differential group delay between $L P_{01}$ and $L P_{02}$ modes. For average power levels $<0 \mathrm{dBm}$ (Fig 2. (a)), both modes propagated in a linear regime. The $\mathrm{LP}_{01}$ mode shows the expected adiabatically compressed $\mathrm{N}=1$ soliton at a launched average power of $3.4 \mathrm{dBm}$ assuming a propagation loss of $0.2 \mathrm{~dB} / \mathrm{km}$. For $7 \mathrm{dBm}$ higher power soliton splitting occurs (Fig. 2 (c)) and a clear higher order $(\mathrm{N}=2)$ soliton at $13.4 \mathrm{dBm}$ is observed (Fig. 2 (d)). One would expect that when moving to higher powers all independent modes would share the soliton-like propagation of the $\mathrm{LP}_{01}$. However, the higher order mode $\mathrm{LP}_{02}$ did not demonstrate the same behaviour, with unexpected features appearing even at lower powers.

A numerical model was used to predict the behaviour of both modes by solving the nonlinear Schrödinger equation using a split-step Fourier method, taking into account the chromatic dispersion of each mode, loss and nonlinearities, but excluding cross-mode coupling and delayed nonlinear responses. To improve the accuracy of the numerical model the experimental phase and amplitude information from input pulses, measured by the frequency discriminator technique, were seeded into the numerical model. The simulated results are also shown in Fig. 2 (red), which confirms that for the $\mathrm{LP}_{01}$, the observed results are consistent with the low mode coupling soliton-like pulses observed in typical $50 \mu \mathrm{m}-$ MMF [6]. For this mode, the observed soliton propagation is also similar to that of solitons in SMF [11], where a pulse width minimum is achieved at two power levels $\sim 10 \mathrm{~dB}$ apart, suggesting that there is no power dependent mode coupling for this mode [12] and agreement between the experimental results and the numerical model is clear. However, for the higher order mode $\left(\mathrm{LP}_{02}\right)$, where the model predicts a nonlinear response similar to that of $\mathrm{LP}_{01}$, soliton propagation is less clear. We believe that this is due to a combination of linear and nonlinear interactions between the modes, such as power dependent mode-coupling and the presence of cross-mode coupling, not included in the model. It is believed that the $\mathrm{LP}_{02}$ mode is probably affected by linear mode coupling (Fig 2. (b)) at first, but for higher powers (Fig. 2 (c-d)) nonlinear mode coupling may also occur, evident by the non-symmetrical pulse shapes of the $\mathrm{LP}_{02}$ mode for high powers..

\section{Conclusions}

This paper shows the first demonstration that $\mathrm{N}=1$ and $\mathrm{N}=2$ adiabatic solitons may exist in an individual mode on a few mode optical fibre. The fundamental mode $\left(\mathrm{LP}_{01}\right)$ shows behaviour similar to that of a soliton in SMF, but the higher order mode showed more complex behaviour, indicating that power dependent mode coupling along with other effects such as mode coupling are present.

\section{REFERENCES}

[1] A. D. Ellis, et al., Journal of Lightwave Technology, vol. 28, pp. 423-433, Feb 2010.

[2] S. Randel et al., OFC 2012, PDP5C.5

[3] F. Ferreira et al., IEEE Photonics Technology Letters, Vol. 24, No. 4, Feb 15, 2012

[4] F. Poletti et al., J. Opt. Soc., Vol. 25, No. 10, Oct 2008

[5] B. Crosignani et al., Optics Letters, Vol. 6, No.7, Jul 1981

[6] K. S. Chiang, Optics Letters, Vol.17, No. 5, Mar 1, 1992

[7] D. Marcuse, “Theory of Dielectric Optical Waveguides”, Academic Press, 1974

[8] P. L. Baldeck et al., Optics Letters, Vol. 12, No. 8, Aug 1987

[9] R. Watts et al., Optics Communications, vol. 285, no. 8, 2012,

[10] K. DeLong et al., J. Opt. Soc. Am. B, vol. 11, pp. 22062215, 1994

[11] H. A. Haus, Proceedings of the IEEE, Vol. 81, No. 7, Jul 1993

[12] M. Mitchell, Physical Review Letters, Vol. 80, No. 21, May 25, 1998 